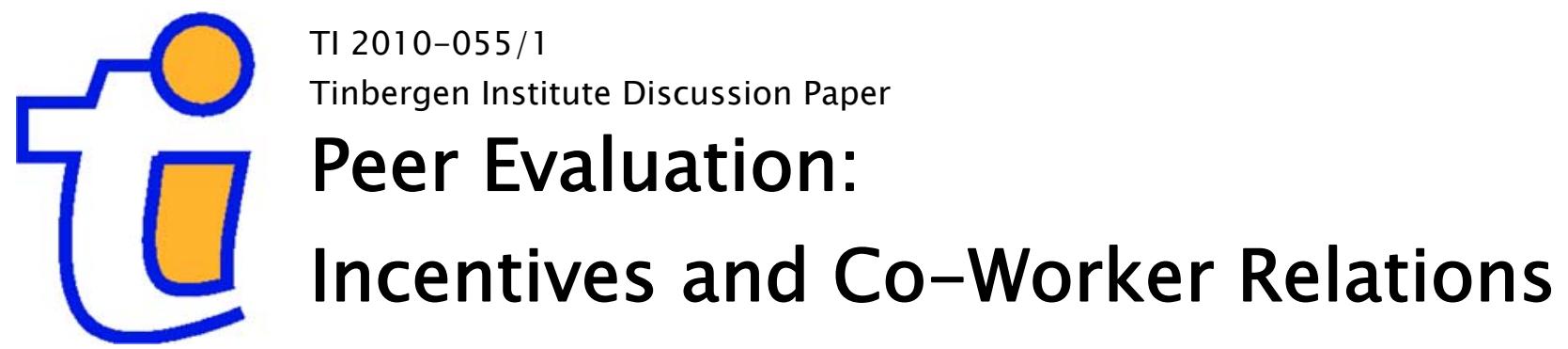

Joeri Sol

Department of Economics, Erasmus University Rotterdam, and Tinbergen Institute. 


\section{Tinbergen Institute}

The Tinbergen Institute is the institute for economic research of the Erasmus Universiteit Rotterdam, Universiteit van Amsterdam, and Vrije Universiteit Amsterdam.

Tinbergen Institute Amsterdam

Roetersstraat 31

1018 WB Amsterdam

The Netherlands

Tel.: +31(0)205513500

Fax: $+31(0) 205513555$

Tinbergen Institute Rotterdam

Burg. Oudlaan 50

3062 PA Rotterdam

The Netherlands

Tel.: + $31(0) 104088900$

Fax: $+31(0) 104089031$

Most TI discussion papers can be downloaded at http://www.tinbergen.nl. 


\title{
Peer Evaluation: Incentives and Co-worker Relations*
}

\author{
Joeri Sol ${ }^{\dagger}$
}

May 28, 2010

\begin{abstract}
In many workplaces co-workers have the best information about each other's effort. Managers may attempt to exploit this information through peer evaluation. I study peer evaluation in a pure moral hazard model of production by two limitedly liable agents. Agents receive a signal about their colleague's effort level, and are asked to report it to the principal. The principal may give an individual bonus for the receipt of a positive evaluation by a colleague, which stimulates effort as long as signals are revealed truthfully. A cost of lying ascertains that there can be truthful revelation. I show that interpersonal relations between colleagues constrain the bonus for receiving a positive evaluation in order to keep evaluations truthful. Still, the principal will always include such a bonus in the optimal contract, and possibly complement it with a team bonus. Co-worker relations have non-monotic effects on profits in the optimal contract.
\end{abstract}

JEL-codes: D86, J33, M50.

Keywords: peer evaluation, peer appraisal, incentive contracts, co-worker relations, likeability bias.

${ }^{*}$ I gratefully acknowledge comments and suggestions by Josse Delfgaauw, Robert Dur, Amihai Glazer, Vladimir Karamychev, Navin Kartik, Paul Steffens, and seminar participants at Erasmus University and at University of Amsterdam.

†Tinbergen Institute, Erasmus University Rotterdam. E-mail: jsol@ese.eur.nl. 


\section{Introduction}

In many employment relations managers lack information about employees' individual effort or performance; e.g. in highly interdependent work teams, with work on location, or in case of specialists. In an attempt to obtain a more complete picture of employee performance, many firms have turned to multisource feedback, in business better known as $360^{\circ}$ evaluations. A $360^{\circ}$ evaluation can include performance assesments by subordinates, peers, supervisors, customers, or other stakeholders. According to survey data, about 90\% of Fortune 1000 firms use some form of multisource feedback, often including evaluation by peers (Edwards and Ewen 1996). In a different survey, among member firms of the American Management Association, a fifth of the 750 responding companies reports that they use peer evaluation. In $90 \%$ of these companies peer evaluation partially determines personnel decisions regarding promotions and performance pay (Bohl 1996). A similar percentage of peer evaluation usage is found in another sample of 280 USbased firms (Antonioni 1996).

Evaluation by peers has intuitive appeal when employees have valuable information about one another's performance. According to the General Social Survey this prerequisite is often met: Around $70 \%$ of the respondents reports to observe the behavior of colleagues with a score of 8 out of 10 or higher (Freeman et al. 2008). However, when seen from an economic theory perspective, the widespread use of peer evaluation is less obvious. A major concern is that employees may benefit from providing the employer with invalid performance evaluations of their colleagues. For instance, employees can provide invalid ratings so as to help their friends, hurt their competitors, or 'game the system' through collusion (Edwards and Ewen 1996 and Kozlowski et al. 1998). This paper develops a formal agency model so as to study when and how peer evaluation should be used under different intensities of co-worker relations.

I study peer evaluation in a model of team production by two homogeneous agents, who are protected by limited liability. The production suffers from a pure moral hazard problem, i.e., the principal is unable to distinguish individual inputs, and can only offer a team bonus for output. A team bonus results in inefficiently low effort levels, since limited liability makes the budget breaking solution infeasible. Agents receive a signal about their colleague's effort provision. The principal may attempt to improve upon the team bonus by making use of the agents' information through peer evalu- 
ation. Agents are asked to report the signal about their colleague's effort, where this evaluation determines whether the colleague receives a bonus. As a starting point I assume ideal circumstances for these peer evaluations: Agents have an aversion towards lying about their signal, leading them to make truthful evaluations. The bonus for the receipt of a positive peer evaluation motivates agents to exert effort so as to increase the likelihood that their co-worker receives a positive signal and rates performance accordingly. Under these ideal circumstances, peer evaluation outperforms a team bonus; in fact, peer evaluation performs as well as individual incentives would.

Next, I allow for interpersonal relations between colleagues, either good or bad. Social preferences can give agents an incentive to lie about their signal. If the utility from a bonus to a friend outweighs the cost of lying, agents with good co-worker relations give positive evaluations regardless of their signal. Likewise, agents in bad relationships may begrudge a bonus to their colleague and lie upon receiving a positive signal. Invalid peer evaluations also provide incentives for effort, through internalization of a colleague's lying costs. Profits, however, are usually higher when peer evaluations remain truthful. The principal can ensure truthful evaluation by adjusting the bonus in peer evaluation downward, such that co-worker relations do not affect the evaluation decision. Social preferences, thereby, constrain the effectiveness of peer evaluation. Nevertheless, the optimal contract always includes peer evaluation. The principal will complement incentives for effort by including a team bonus in case peer evaluation becomes severely constrained. The effectiveness of the team bonus increases with better co-worker relations, as co-worker relations moderate incentives to free-ride on a colleague. Peer evaluation suffers from more pronounced co-worker relations, through the earlier discussed likeability bias. Taken together, co-worker relations have non-monotonic effects on profits.

Despite the widespread use of peer evaluation, there are few empirical studies on its effectiveness. The success of peer evaluation programs is difficult to determine, since objective performance measures are often lacking, motivating peer assesment to start with. Peiperl (1999) is one of the few empirical studies that looks into success factors of peer evaluation in business. Within a multinational service-sector organization managers were asked to identify units with (un)successful peer evaluation. Successful peer evaluation was positively correlated with a high interdependency of work within and across units, group components in incentives, the perceived quality of the ratings, and its integration with rewards. In addition, peer evaluation was 
found to be less successful in units that had a very positive group culture, of which Peiperl says: "an unexpected result that bears some consideration" (p. 445). The likeability bias I study in this paper can explain this observation.

The results of this paper, that better co-worker relations erode peer evaluation, while they strengten the effectiveness of a team bonus, are largely in line with findings by Towry (2003). In a laboratory experiment, she stud-

ies how the effectiveness of these two incentive schemes are influenced by a team identity manipulation. In this experiment, participants either receive a compensation based on a colleague's report about performance, i.e. peer evaluation, or they receive team bonus with punishment opportunities in a second stage. Towry finds that the peer evaluations are undermined by strong team identity, because team members became more likely to cover for each other's shirking behavior. The team incentive, on the other hand, was more effective in this strong team identity treatment, as these teams achieved higher levels of cooperation, without a difference in punishment behavior.

The paper is organized as follows. The next section gives a brief overview of the related literature. Section 3 describes the set-up of the model, followed by the analysis in Section 4. Section 5 contains concluding remarks.

\section{Related literature}

In a pioneering paper, Ma (1988) shows that there exist mechanisms where it is in the agents' best interest to report shared information truthfully. Ma considers a principal who is unable to observe the inputs of a team of agents, while agents observe each others' actions perfectly. The principal can implement first-best effort by asking one agent to report the effort choices of all team members, and giving other agents the option to challenge this report. There exists a lottery for which the option to challenge a report is exercised if and only if the report contains a lie. Since other agents will always challenge a false report, it is optimal for the reporting agent to state the chosen actions truthfully. The principal, thereby, retrieves the agents' information on individual inputs, which enables him to implement first-best effort.

This paper differs from the set-up by Ma in the agents' ability to observe each others' action: Instead of perfect observability, or an assumption that signals are sufficient statistics for effort (Fischer and Hughes 1997), I assume that agents get a coarse signal of a colleague's performance. In the set-up I consider, the principal does not benefit from asking agents to report their 
own effort choice in addition to the signal about a co-worker's effort. The coarse signals do not allow colleagues to verify the stated reports perfectly, hence the principal cannot play off agents' reports against each other. As a consequence, agents have no incentive to report their effort truthfully, rendering the mechanism above infeasible. In many work settings, e.g. within health care, consultancy, or academics, the performance assesment by peers is relevant, yet it is rarely based on perfect information about effort.

Instead of interpersonal relations, Barron and Gupte (2009) focus on other limitations of peer evaluation. The authors introduce harmful consequences of negative peer appraisals, modelled in two ways: First, negative appraisals make agents less productive through a drop in team cohesion. Second, agents experience a loss of utility due to peer pressure. Barron and Gupte show that, in a Ma-type of lottery for truthtelling, agents need a higher compensation to report shirking by a colleague. ${ }^{1}$ Peer evaluation is no longer guaranteed to be profitable under these adverse effects of a negative appraisal, instead profitability depends on the quality of signals among peers.

Marx and Squintani (2009) also analyse a pure moral hazard problem in team production, where agents can be given the task to evaluate each other. Without peer evaluation, the combination of unobservable individual effort choices, and an inability to punish individuals without proof of shirking behavior, results in low output. The principal can achieve the first-best outcome with a contract that demands both high effort and peer monitoring, notwithstanding that monitoring is costly. The peer monitoring gives an incentive to provide effort, as the principal can now punish upon observing low output. Punishment is possible, since peer monitoring provides verifiable evidence of shirking, or in case evidence is lacking, agents failed to monitor. The principal will not punish agents for not monitoring with high output, because he cannot distinguish between non-monitoring and the absence of evidence for shirking. Therefore, the agents exert effort and refrain from monitoring in equilibrium, leading to the first-best. The authors show that their results are sensitive to the monitoring technology.

\footnotetext{
${ }^{1}$ The lottery offered in Barron and Gupte (2009) plays off the signal that a co-worker receives against a signal the principal has, while Ma (1988) only uses the information that the agents share.
} 


\section{The model}

I consider a principal who employs two homogeneous agents, denoted by $i$ and $j$, and maximizes the following profit function:

$$
\pi=\varphi\left(e_{i}, e_{j}\right) Q_{H}-\sum_{i}\left[w_{L i}+\varphi\left(e_{i}, e_{j}\right) \Delta w+m_{j i} B_{i}\right], \text { where } i \neq j .
$$

The principal's profit equals output minus wages. Output can be high or low, where high output has value $Q_{H}$ and low output is set to zero. The probability of high output depends on the effort of both agents, $\varphi\left(e_{i}, e_{j}\right)=$ $e_{i}+e_{j} .{ }^{2}$ The principal only observes the output level that is realized, i.e., effort choices are unobservable. Wages can contain a base wage, $w_{L i}$, a team bonus for high output, $\Delta w$, and a bonus for the receipt of a positive peer evaluation, $B_{i}$. Wages are subject to a limited-liability constraint: The wage cannot be negative in any state of the world. This gives the following limitedliability constraints:

$$
w_{L i} \geq 0, w_{L i}+B_{i} \geq 0, w_{L i}+\Delta w \geq 0, w_{L i}+B_{i}+\Delta w \geq 0 .
$$

Agents maximize the following utility function:

$$
U_{i}=w_{L i}+\varphi\left(e_{i}, e_{j}\right) \Delta w+m_{j i} B_{i}-C\left(e_{i}\right)-\gamma\left|m_{i j}-s_{i j}\right|+\alpha U_{j} .
$$

Utility is increasing in the wage and decreasing with costs of effort. For convenience, I assume quadratic costs of effort: $C\left(e_{i}\right)=\frac{1}{2} e_{i}^{2}$. The principal asks agents to evaluate the performance of their colleague: Agent $i$ sends an evaluation message to the principal about agent $j$ 's performance, denoted by $m_{i j}$. Agents can base their evaluation on a signal they receive about their colleague's effort level. The signal, $s_{i j} \in\{0,1\}$, takes on zero when agent $i$ perceives $j$ 's effort is below some standard and one if $j$ performed well according to $i$. The probability of a high signal depends on the colleague's effort, $\operatorname{Pr}\left(s_{i j}=1\right)=p\left(e_{j}\right)=e_{j}$. I assume that there are some costs of deviating from one's signal, or $\gamma>0$. These costs can stem from the time and energy spent to misrepresent information (Kartik 2009) and many people

\footnotetext{
${ }^{2}$ The assumption of a probabilistic team output is not essential, it is important that individual inputs are unobservable to the principal. It does simplify the analysis by the reduction of the number of possible team bonuses. Further, it creates the need for a restriction on $Q_{H}$, or on the cost of effort, such that high output with certainty is not preffered over the optimal contract.
} 
bear a psychological costs of lying (Gneezy 2005, Hurkens and Kartik 2009, Fischbacher and Heusi 2008). The last term in the utility function captures social preferences in the workplace. A positive $\alpha$ captures altruism, whereas a negative $\alpha$ represents feelings of spite. As a consequence of the homogeneity assumption, $\alpha$ can also be interpreted as the quality of a relationship between colleagues. Substitution of $U_{j}$ into (3) gives:

$$
U_{i}=\frac{1}{1-\alpha^{2}}\left\{\begin{array}{c}
w_{L i}+\varphi\left(e_{i}, e_{j}\right) \Delta w+m_{j i} B_{i}-C\left(e_{i}\right)-\gamma\left|m_{i j}-s_{i j}\right| \\
+\alpha\left[w_{L j}+\varphi\left(e_{i}, e_{j}\right) \Delta w+m_{i j} B_{j}-C\left(e_{j}\right)-\gamma\left|m_{j i}-s_{j i}\right|\right]
\end{array}\right\},
$$

where $\alpha$ is restricted to $-1<\alpha<1$, to ensure an interior solution. ${ }^{3}$

The timing of the game is as follows: First, there is a contracting stage, where the agents can accept or reject the offered contract. Next, each agent chooses effort. Finally, the agents receive a signal about their co-worker's effort level and send an evaluation message to the principal, after which output is realized and the contracts are executed.

\section{Analysis}

In a situation without peer evaluation, the principal can only use the team bonus to stimulate effort provision. The team bonus suffers from a wellknown internalization problem; as the agents' costs of effort are private, while the benefits are not fully internalized, the free-rider problem arises. Still, the principal can achieve first-best profits with a team bonus when budget breaking is possible (Holmstrom, 1982). The principal pays the full marginal product to both agents, and achieves first-best profits through a negative base wage. Budget breaking is not feasible here, as agents are assumed to be limitedly liable. Hence, a team bonus cannot implement first-best effort. Next, let us see whether it is possible to improve upon the team bonus by peer evaluations.

The game is solved backwards, starting with the peer evaluation stage. As a consequence of the binary signal, the principal believes at best two

\footnotetext{
${ }^{3}$ The term $\frac{1}{1-\alpha^{2}}$ is like a social multiplier of utility. In case of positive relations, agent $i$ not only enjoys the utility of $j$, but also that $j$ values $i$ 's well-being. In case of bad relations, $j$ 's utility enters as a cost in $U_{i}$. However, $i$ 's fortune also makes $j$ less well off, so that $i$ 's 'private' utility is again amplified. Still, if agents are identical, the latter effect is second-order, and agents are worse off with bad co-worker relations. At the end of section 4, I will discuss comparative statics with respect to $\alpha$.
} 
messages in equilibrium, $m_{i j} \in\{0,1\} .{ }^{4}$ Agents choose the evaluation message that maximizes their utility. A low evaluation brings a cost of lying in case the received signal was high. Similarly, giving a high evaluation brings a cost of lying when the signal was low, and it leads to a bonus for the colleague. The agent's evaluation remains truthful $\left(m_{i j}=s_{i j}\right)$, as long as:

$$
U_{i}\left(e_{i}, 0\right)-U_{i}\left(e_{i}, 1\right)=\frac{\gamma-\alpha B_{j}}{\left(1-\alpha^{2}\right)} \geq 0, \text { or } \alpha \leq \frac{\gamma}{B_{j}},
$$

for $s_{i j}=0$, and with $s_{i j}=1$ :

$$
U_{i}\left(e_{i}, 1\right)-U_{i}\left(e_{i}, 0\right)=\frac{\gamma+\alpha B_{j}}{\left(1-\alpha^{2}\right)} \geq 0, \text { or } \alpha \geq-\frac{\gamma}{B_{j}}
$$

Obviously, without social preferences (i.e., $\alpha=0$ ), the assumption of lying costs leads to a truthful revelation of one's signal. However, once we allow for co-worker relations, agents may have an incentive to lie. Namely, the bonus for receiving a positive evaluation gives a benefit or cost depending on the agent's social preferences. Agents in good relationships may have an incentive to lie upon receiving a low signal about their colleague's performance: When the utility of a bonus for a friend outweighs the lying costs, agents give each other positive evaluations irrespective of the received signal. Likewise, colleagues in a bad relationship may want to lie in case of a positive signal, as they begrudge the bonus to a disliked colleague. Truthful evaluation is thereby constrained to $-\frac{\gamma}{B} \leq \alpha \leq \frac{\gamma}{B}$; i.e., when agents have weak social preferences, high costs of lying, or there is a small bonus for a positive evaluation. Lemma 1 summarizes an agent's evaluation strategy:

Lemma 1 Agent $i$ 's evaluation strategy $i s: m_{i j}=\left\{\begin{array}{ll}0 & \text { if } \alpha<-\frac{\gamma}{B_{j}} \\ s_{i j} & \text { if }-\frac{\gamma}{B_{j}} \leq \alpha \leq \frac{\gamma}{B_{j}} \\ 1 & \text { if } \alpha>\frac{\gamma}{B_{j}}\end{array}\right.$. The evaluation process is truthful when agents have weak social preferences, high lying costs, or small benefits from a positive evaluation. This translates

\footnotetext{
${ }^{4}$ For other messages, I assume that the principal believes the agent received a low signal, and awards no bonus.
} 
into a constraint on the bonus for receiving a positive peer evaluation under which peer evaluations remain truthful:

$$
B \leq \frac{\gamma}{|\alpha|}
$$

In the preceding stage agents choose their effort levels. The first-order condition for effort is the following:

$$
\left(1-\alpha^{2}\right) \frac{d U_{i}}{d e_{i}}=(1+\alpha) \varphi_{e_{i}}(\cdot) \Delta w-C_{e_{i}}(\cdot)+\frac{d U_{i}}{d E\left(s_{j i}=1\right)} \frac{d E\left(s_{j i}=1\right)}{d e_{i}}=0 .
$$

Effort provision increases with the team bonus, while it decreases with the marginal costs of effort. The team bonus for high output motivates agents to exert effort, in order to increase its likelihood. The team bonus also affects the income of a colleague. The latter effect can be an additional benefit or costs of effort depending on agent $i$ 's social preferences. The team bonus provides stronger incentives when agent $i$ is altruistic towards $j$, while effort provision is supressed when $i$ is spiteful towards $j$. The last term in the first-order condition captures incentives for effort from peer evaluation. The expectation that a co-worker receives a positive signal is increasing in effort, $\frac{d E\left(s_{j i}=1\right)}{d e_{i}}=1$, and a change in the co-worker's signal affects utility as follows:

$$
\frac{d U_{i}}{d E\left(s_{j i}=1\right)}\left\{\begin{array}{ll}
-\alpha \gamma & \text { if } m_{i j}=0 \\
B_{i} & \text { if } m_{i j}=s_{i j} \\
\alpha \gamma & \text { if } m_{i j}=1
\end{array} .\right.
$$

Combined, these terms give two incentive effects. First, in case of truthful peer evaluation, effort is stimulated by the peer evaluation bonus. Agents desire to increase the likelihood that their colleague receives a positive signal and rates performance accordingly, such that the bonus is obtained. Second, under non-truthful peer evaluation there is also an incentive for effort, despite that the signal about a co-worker's performance is ignored in the evaluation decision. Altruistic agents wish to increase the probability that their colleague receives a positive signal, such that the expected lying costs for this colleague are lower. Likewise, spiteful agents can increase the lying costs of a colleague by increasing the probability of a positive signal. The incentives from non-truthful peer evaluation are weaker than those of the maximum bonus under truthful peer evaluation:

$$
B=\frac{\gamma}{|\alpha|}>|\alpha| \gamma \text { as }|\alpha|<1
$$


For non-truthful peer evaluation the bonus for receiving a positive evaluation does not give incentives for effort itself, however the bonus should be sufficiently large to give rise to non-truthful peer evaluation strategies. ${ }^{5}$ It is evident that non-truthful peer evaluations is unattractive to the principal when co-worker relations are good, as the incentives are weaker and the higher $B$ always needs to be paid. In case of bad co-worker relations, the non-truthful equilibrium has potential to be more profitable, as the peer evaluation bonus is never paid out. Nonetheless, the principal often achieves higher profits with truthful peer evaluation, for which a sufficient condition is:

$$
\alpha \gamma>-\frac{1}{2}(1+\alpha) Q_{H}
$$

see the appendix for the proof. In case this condition does not hold, the principal can exploit a bad relationship between agents by setting a high peer evaluation bonus, which is never paid. As described before, agents have an incentive to work, because they can increase the expected lying costs of a disliked colleague. In the remainder of this paper I will assume that (6) holds, since the practical relevance of this non-truthful equilibrium is to my opinion limited. Consequently, we can focus on the optimal effort choice under truthful peer evaluation. In combination with the assumptions on $\varphi(\cdot)$ and $C(\cdot)$, the first-order condition for effort implies that agent $i$ 's optimal effort under truthful peer evaluation equals:

$$
e_{i}=(1+\alpha) \Delta w+B_{i}
$$

Summarizing the above: Effort can be stimulated through a team bonus and a bonus for receiving a positive peer evaluation. The effectiveness of the team bonus depends on an agent's social preferences. The free-rider problem, inherent to a team bonus, is less severe when co-worker relations are good. The free-rider problem is exacerbated for bad co-worker relations, as agents begrudge their colleague the fruits of their labour. Further, a bonus for the receipt of a positive peer evaluation stimulates effort provision, because effort influences a colleague's expected evaluation message under truthful peer evaluation.

\footnotetext{
${ }^{5}$ Note that, peer evaluation without a bonus, or $B_{i}=0$, gives no incentive for effort. Developmental peer evaluation, or peer evaluation without compensation, is a much used tool, but without value in the setting I study. Recently, Gupte (2009) presents a model of developmental peer evaluation, where peer evaluation is used as an input to determine a suitable training.
} 
Finally, the principal sets the profit maximizing contracts subject to the incentive compatibility constraints (7), the limited-liability constraints (2), and the constraint for truthful peer evaluation (4). Naturally, the principal does not need to abide to this last constraint (4). The inclusion of this constraint helps to characterize the optimal contract, but is innocuous, as the principal cannot do better with non-truthful peer evaluation when (6) holds. Together, this gives the principal's optimization problem:

$$
\begin{aligned}
\max _{w_{L i}, \Delta w, B_{i}, B_{j}} \pi & =\varphi(\cdot)\left[Q_{H}-2 \Delta w\right]-w_{L i}-w_{L j}-E\left(m_{i j}\right) B_{j}-E\left(m_{j i}\right) B_{i}, \\
\text { s.t. } w_{L i} & \geq 0, w_{L i}+B_{i} \geq 0, w_{L i}+\Delta w \geq 0, w_{L i}+B_{i}+\Delta w \geq 0, B_{i} \leq \frac{\gamma}{|\alpha|},
\end{aligned}
$$

where the last limited-liability constraint is satified by a combination of the former constraints. The first limited-liability constraint will bind, as there is no incentive for effort from the base wage. Hence, we get $w_{L i}=0$, and the optimization problem is reduced to:

$$
\begin{aligned}
\max _{\Delta w, B_{i}, B_{j}} \pi & =\varphi(\cdot)\left[Q_{H}-2 \Delta w\right]-w_{L i}-w_{L j}-E\left(m_{i j}\right) B_{j}-E\left(m_{j i}\right) B_{i}, \\
\text { s.t. } \Delta w & \geq 0, B_{i} \geq 0, B_{i} \leq \frac{\gamma}{|\alpha|}
\end{aligned}
$$

leading to the second-best contract described in Proposition 1:

Proposition 1 The optimal contract under limited liability is characterized by:

$$
\begin{aligned}
& B_{i}=B_{j}=\frac{1}{2} Q_{H} \text { and } \Delta w=0, \text { if } \gamma \geq \frac{1}{2}|\alpha| Q_{H}, \\
& B_{i}=B_{j}=\frac{\gamma}{|\alpha|} \text { and } \Delta w=0, \text { if } \frac{(1+\alpha)|\alpha| Q_{H}}{3+\alpha} \leq \gamma \leq \frac{1}{2}|\alpha| Q_{H}, \\
& B_{i}=B_{j}=\frac{\gamma}{|\alpha|} \text { and } \Delta w=\frac{Q_{H}}{4}-\frac{(3+\alpha) \gamma}{4(1+\alpha)|a|}, \text { if } \gamma<\frac{(1+\alpha)|\alpha| Q_{H}}{3+\alpha}(\text {. } C)
\end{aligned}
$$

The proof can be found in the appendix. The optimal contract always includes a bonus for receiving a positive peer evaluation. The principal can ensure peer evaluation remains truthful, by satisfying constraint (4). This allows the principal to offer individual incentives for effort through $B_{i}$, even 
though he does not observe the individual contributions himself. Hence, unlike the team bonus, the peer evaluation bonus does not suffer from an internalization problem, which makes it a more cost-effective way to stimulate effort.

Part (A) of Proposition 1 shows the optimal contract under ideal circumstances for peer evaluation, i.e. sufficiently high cost of lying or weak social preferences. In this situation, the peer evaluation bonus performs as well as providing the agents with individual incentives under limited liability. Smaller lying costs or more pronounced social preferences constrain the peer evaluation bonus at some point, described by contract (B). Eventually, the principal will find it optimal to complement incentives for effort with a team bonus, as indicated by contract (C) in Proposition 1.

The comparative statics of $\alpha$ and $\gamma$ with respect to the peer evaluation bonus and the team bonus are described by Corollary 1:

Corollary 1 In contracts $(B)$ and $(C)$, the bonus for the receipt of a positive peer evaluation is increasing in lying costs and social preferences that become less pronounced:

$$
\begin{aligned}
& \frac{\partial B}{\partial \gamma}=\frac{1}{|\alpha|}>0 \\
& \frac{\partial B}{\partial \alpha}=-\frac{\alpha \gamma}{|\alpha|^{3}} .
\end{aligned}
$$

In contract $(C)$, the team bonus is decreasing with the costs of lying, and social preferences have an ambiguous effect on the team bonus:

$$
\begin{aligned}
\frac{\partial \Delta w}{\partial \gamma} & =-\frac{\partial B}{\partial \gamma} \frac{(3+\alpha)}{4(1+\alpha)}<0 \\
\frac{\partial \Delta w}{\partial \alpha} & =-\frac{\partial B}{\partial \alpha} \frac{(3+\alpha)}{4(1+\alpha)}+\frac{1}{2(1+\alpha)^{2}} \frac{\gamma}{|\alpha|} .
\end{aligned}
$$

Otherwise, lying costs and social preferences do not affect compensation in the second-best contract.

Corollary 1 shows a negative relation between lying costs and the team bonus. A decrease in the costs of lying paralyzes peer evaluation through the constraint to keep evaluations truthful, which can make it optimal (if lying 


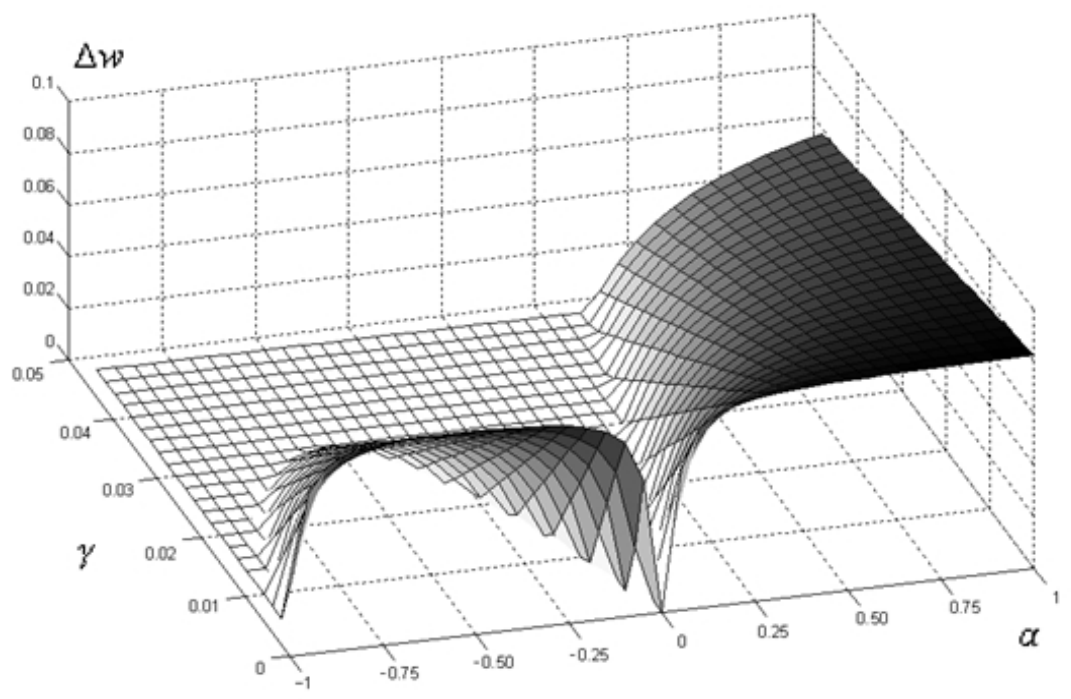

Figure 1: Team bonus in the optimal contract for different $(\gamma, \alpha)$ combinations, with $Q_{H}=\frac{1}{3}$.

costs are sufficiently low) to enhance effort provision with a higher team bonus. Social preferences have a twofold influence on the strength of the team bonus, as can be seen from (9). First, like with lying costs, stronger social preferences constrain the bonus for receiving a positive peer evaluation. Stronger social preferences, whether negative or positive, thereby add to the importance of the team bonus. Second, social preferences also influence the incentive effects of a team bonus, as we already observed in (7). For altruistic agents better co-worker relations strengthen the team bonus by both effects. The two effects are in conflict for bad co-worker relations that become worse, which leads to a decrease of the team bonus at some point for more spiteful agents. The optimal team bonus in the second-best contract is depicted in Figure 1 for different $(\gamma, \alpha)$ combinations, with $Q_{H}=\frac{1}{3}$. Figure 2 and Figure 3 do so for the expected profits and expected utility. ${ }^{6}$ The comparative statics for the expected profits and expected utility can be found in the appendix.

\footnotetext{
${ }^{6}$ The figures are qualitatively the same for other levels of $Q_{H}$. The sufficient condition for optimality of truthful peer evaluation (6) does not hold for some $(\gamma, \alpha)$ combinations in the upper left corners in Figure 1 to 3, in which case the optimal contract may look different.
} 


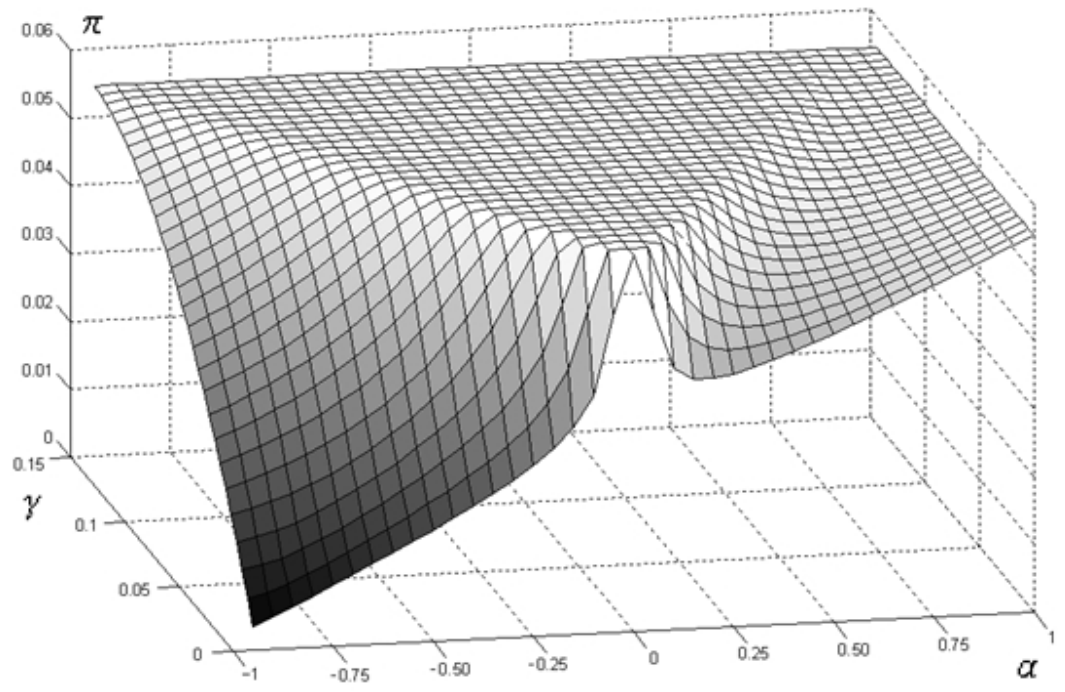

Figure 2: Expected profits under the optimal contract for different $(\gamma, \alpha)$ combinations, with $Q_{H}=\frac{1}{3}$.

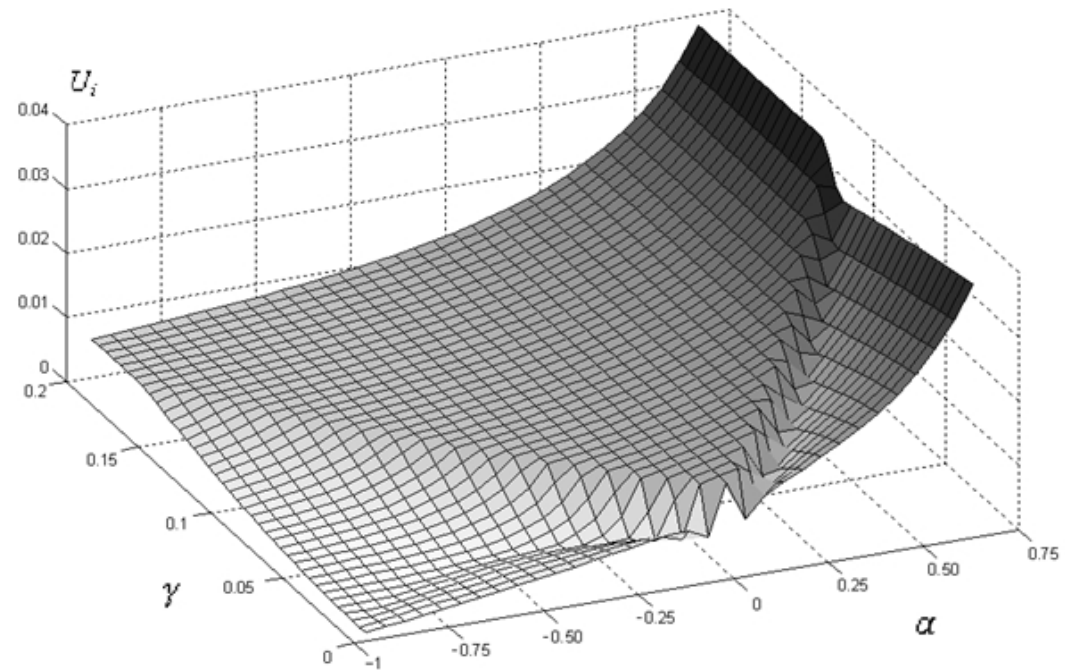

Figure 3: Expected utility in the optimal contract for different $(\gamma, \alpha)$ combinations, with $Q_{H}=\frac{1}{3}$. 
As long as peer evaluation is unrestricted (A), the expected profits are constant. In contract (B), lower lying costs and more pronounced co-worker relations hurt profits, through the smaller incentive effect of the peer evaluations. Finally, in contract $(\mathrm{C})$, the size of the peer evaluation bonus and the team bonus are both influenced by $\gamma$ and $\alpha$. A decrease of the lying costs leads to a shift in the compensation towards the team bonus. This shift hurts profits, as the peer evaluation bonus stimulates effort in a more cost-effective way than the team bonus does, so profits are still increasing in $\gamma$ under contract $(\mathrm{C})$. There is a similar effect of more pronounced co-worker relations, a shift away from the peer evaluation bonus has a negative effect on profits. In addition, better co-worker relations soften the free-rider problem, which has a favorable effect on profits. Profits are increasing in $\alpha$ with bad co-worker relations, as both effects are in the same direction. For good co-worker relations, profits are decreasing in $\alpha$ at first, as the shift away from peer evaluation dominates the effect of a softened free-rider problem. However, the profits pick up again from some point, as the effect on the free-rider problem becomes more prominent for a larger team bonus. Summarizing, under truthful peer evaluation, bad co-worker relations are never good for profits, while good co-worker relations can be bad.

Agents are left with rents due to the limited liability assumption. Lying costs only affect utility through the offered contract, since there is no lying in equilibrium. Therefore, utility is constant in $\gamma$ for unrestricted peer evaluation (A). Co-worker relations, on the other hand, have a direct and positive effect on utility, as already noted in footnote 3 . In contract (B), an increase in the lying costs relaxes the constraint on the peer evaluation bonus, and thereby leads to a higher utility. Similarly, besides the direct effect on utiltiy, co-worker relations affect the size of the peer evaluation bonus. The expected utility is increasing in $\alpha$, except for moderately positive co-worker relations $\left(0<\alpha<\frac{2}{3}\right)$, where the decrease in the peer evaluation bonus outweighs the direct effect of having better co-worker relations. Finally, in contract (C), higher lying costs cause a shift in the optimal compensation towards peer evaluation, as noticed before. This shift away from the team bonus hurts the agents' utility, as the team bonus comes with higher rents. A change in $\alpha$ also has this effect, in addition to the direct effect on utility and the effect on the free-rider problem. The latter two effects make the utility increasing in co-worker relations, whereas the first effect is negative for $\alpha<0$ and can dominate for moderately negative co-worker relations. 


\section{Concluding remarks}

Peer evaluation has intuitive appeal in work environments where co-workers are in the best position to monitor each other. I have studied the possibility to include peer evaluation in performance pay using a model of team production by two limitedly liable agents. The principal only observes the team output, while agents receive a signal about a colleague's effort, allowing for peer evaluation in addition to a team bonus. The combination of a cost of lying about the received signal and interpersonal relations between co-workers determine the agent's evaluation decision. Lying costs lead to a truthful revelation of signals, while the internalization of a bonus to a colleague may cause a bias. Nevertheless, the optimal contract always includes a bonus for receiving a positive peer evaluation, which may be complemented with a team bonus. Truthful peer evaluation has incentive effects, as agents desire to increase the likelihood that their colleague receives a positive signal. Social preferences constrain the bonus for a positive evaluation so as to keep revelation of signals truthful, leading to the testable implication, that incentive effects of peer evaluation diminish with more pronounced social relations between co-workers. A managerial implication of this result is that managers should assign employees that are rather indifferent towards one another to evaluate each other's performance.

There is a large debate in the management literature and organizational psychology whether peer evaluations should only be used for developmental purposes, or whether they can also be used to determine merit pay and promotions (see among others Edwards and Ewen 1996, Coates 1998, Fleenor and Brutus 2001). I contribute to this debate by showing that some concerns of intergrating peer evaluation with performance pay can be overcome. Namely, by setting a smaller bonus for peer evaluation, it is possible to avoid a (dis)likeability bias. In addition, I illustrated that even invalid peer evaluations can have incentive effects. The distinction between developmental peer evaluation and its integration with rewards, however, may be less strict in practice: In case a manager is supposed to serve as a coach, it is unlikely that the information resulting from peer evaluations is neglected in determining promotions and pay (Edwards and Ewen 1996). The consequences of including peer evaluation in performance pay, therefore, deserve more research attention.

There are a number of other factors that may lead to intentional distortions in peer evaluation. Peer evaluation between colleagues in promotion 
tournaments will most likely lead to a downward bias in the evaluation reports, in order to boost one's own chances of winning. Carpenter et al. (2010) provide evidence for this subtle way of sabotaging competitors in a real effort experiment, where the anticipation of sabotage eliminates the incentives to work in this tournament. A special case of peer evaluation is where incumbents determine their future co-workers, or competitors for future promotions, as incumbents may be most capable in evaluating the quality of applicants. Carmichael (1988) showed that in such a setting incumbents discriminate against the best possible candidates, unless the incumbents' future income is ensured, which is sometimes only possible by offering tenure. Another concern is that colleagues may try to collude with their evaluations against the principal in repeated interactions.

The result that a principal always wants to make use of peer evaluations is at odds with its occurance in practice. However, the prerequisite that coworkers have valuable information on employee performance does not apply to all organizations. Further, it is conceivable that peer evaluation has an influence on interpersonal relationships. Dur and Sol (2010) found that, under the assumption of conditionally altruistic or reciprocal preferences, externalities in wages may motivate people to invest in their relationships with colleagues. In line with this, peer evaluation may be a good reason to invest in the relationship with a colleague that evaluates one's performance, so as to increase the likelihood of a positive evaluation by this colleague. By this logic the effectiveness of peer evaluation may deteriorate eventually. In combination with some of the additional concerns named above, this offers an explanation for the gap between the theoretical prediction and the prevalence of peer evaluations in practice. 


\section{Appendix}

\section{Proof of Proposition 1}

The agents' effort choices $(7)$ are substituted into $\varphi(\cdot)$ and $E\left(m_{i j}\right)=p(\cdot)$, which gives the following Kuhn-Tucker conditions to the principal's optimization problem (8):

$$
\begin{aligned}
\frac{d \pi}{d \Delta w} & =2(1+\alpha) Q_{H}-8(1+\alpha) \Delta w-(3+\alpha) B_{i}-(3+\alpha) B_{j}+\lambda_{1} \leq 0 \\
\Delta w & \geq 0, \Delta w \frac{d \pi}{d \Delta w}=0, \lambda_{1} \geq 0, \lambda_{1} \Delta w=0, \\
\frac{d \pi}{d B_{i}} & =Q_{H}-(3+\alpha) \Delta w-2 B_{i}+\lambda_{2}-\lambda_{3} \leq 0, B_{i} \geq 0, B_{i} \frac{d \pi}{d B_{i}}=0, \\
\lambda_{2} & \geq 0, \lambda_{2} B_{i}=0, B_{i}-\frac{\gamma}{|\alpha|} \leq 0, \lambda_{3} \geq 0, \lambda_{3}\left[B_{i}-\frac{\gamma}{|\alpha|}\right]=0 \\
\frac{d \pi}{d B_{j}} & =Q_{H}-(3+\alpha) \Delta w-2 B_{j}+\lambda_{4}-\lambda_{5} \leq 0, B_{j} \geq 0, B_{j} \frac{d \pi}{d B_{j}}=0, \\
\lambda_{4} & \geq 0, \lambda_{4} B_{i}=0, B_{j}-\frac{\gamma}{|\alpha|} \leq 0, \lambda_{5} \geq 0, \lambda_{5}\left[B_{j}-\frac{\gamma}{|\alpha|}\right]=0 .
\end{aligned}
$$

We look for a solution where $B_{i}=B_{j}$, as agents are homogeneous. Further, as the constraints on $B_{i}=B_{j}$ can never bind at the same time, the cases that need be checked are reduced to six. (i) none of the constraints is binding, (ii) $\Delta w=0$, and $B_{i}=B_{j}=0$, (iii) $\Delta w>0$ and $B_{i}=B_{j}=0$, (iv) $\Delta w=0$ and constraints on $B_{i}=B_{j}$ are non-binding, (v) $\Delta w>0$ and $B_{i}=B_{j}=\frac{\gamma}{|\alpha|}$, and (vi) $\Delta w=0$ and $B_{i}=B_{j}=\frac{\gamma}{|\alpha|}$.

The first three cases cannot be part of the optimal contract: (i) $\lambda_{i}=0$, for which the first-order conditions solve for $\Delta w=\frac{Q_{H}}{1-\alpha}$, and $B_{i}=B_{j}=$ $-\frac{(1+\alpha) Q_{H}}{1-\alpha}<0$, which contradicts $B_{i} \geq 0$. (ii) $\Delta w=B_{i}=B_{j}=0$, contradicts $\lambda_{1} \geq 0$ and $\frac{d \pi}{d \Delta w} \leq 0$ being valid at the same time. (iii) $\frac{d \pi}{d \Delta w}=0, \lambda_{1}=0$, and $B_{i}=B_{j}=0$ give $\Delta w=\frac{1}{4} Q_{H}$, but also gives: $\frac{d \pi}{d B_{i}}=\frac{d \pi}{d B_{j}}=\frac{1}{4}(1-\alpha) Q_{H}+\lambda_{2,4}$, which contradicts $\frac{d \pi}{d B_{i}}=\frac{d \pi}{d B_{j}} \leq 0$, as $-1<\alpha<1$. The next three cases jointly characterize the optimal contract: (iv) $\frac{d \pi}{d B_{i}}=\frac{d \pi}{d B_{j}}=0$ and $\lambda_{2}=$ $\lambda_{3}=\lambda_{4}=\lambda_{5}=0$ give $B_{j}=\frac{1}{2} Q_{H}$, all conditions are satisfied as long as $\frac{\gamma}{|\alpha|} \geq \frac{1}{2} Q_{H} \geq 0$, otherwise $B_{i}$ will hit either constraint, which leads to 
contradiction. This case leads to the first line in Proposition 1. (v) $\frac{d \pi}{d \Delta w}=0$, and $\lambda_{1}=0$ gives $\Delta w=\frac{1}{4} Q_{H}-\frac{(3+\alpha) \gamma}{4(1+\alpha)|a|}$, all conditions are satisfied as long as $\gamma<\frac{(1+\alpha)|\alpha| Q_{H}}{3+\alpha}$ otherwise $\Delta w \leq 0$ which gives a contradiction. This case gives the third line in Proposition 1. (vi) $\frac{d \pi}{d \Delta w} \leq 0$, and $\lambda_{1} \geq 0$ give $\gamma \geq \frac{(1+\alpha)|\alpha| Q_{H}}{3+\alpha}$, otherwise the two cannot be satisfied simultaneously, and $\frac{d \pi}{d B_{i}}=\frac{d \pi}{d B_{j}}=0$ together with $\lambda_{2}=\lambda_{4}=0$, and $\lambda_{3}=\lambda_{5} \geq 0$ give that $\frac{\gamma}{|\alpha|} \leq \frac{1}{2} Q_{H}$ otherwise a contradiction follows. This gives the second line in Proposition 1 and completes the characterization of the optimal contract.

To complete the proof of the optimal contract we need to compare the profits under truthful peer evaluation with the profits of peer evaluation that is not truthful. For non-truthful peer evaluation under good co-worker relations $\left(m_{i j}=1\right)$, the principal always has to pay a higher peer evaluation bonus, while incentives are weaker. Therefore, it is evident that this decreases the principal's profits. The non-truthful evaluation strategy for bad co-worker relations $\left(m_{i j}=0\right)$ seems more promising, as the principal never has to pay the peer evaluation bonus. The size of the peer evaluation bonus therefore does not matter for profits, it should only be sufficiently high so as to give rise to the non-truthful peer evaluation strategy. The principal still needs to find the optimal team bonus. For $m_{i j}=0$ the agent's optimal effort is:

$$
e_{i}=(1+\alpha) \Delta w-\alpha \gamma
$$

leading to:

$$
\frac{d \pi}{d \Delta w}=(1+\alpha)\left[Q_{H}-4 \Delta w\right]+2 \alpha \gamma=0,
$$

an optimal team bonus under $m_{i j}=0$ :

$$
\begin{aligned}
& \Delta w=\frac{1}{4} Q_{H}+\frac{\alpha \gamma}{2(1+\alpha)} \text { if } \alpha \gamma>-\frac{1}{2} Q_{H}(1+\alpha), \\
& \Delta w=0 \text { if } \alpha \gamma<-\frac{1}{2} Q_{H}(1+\alpha)
\end{aligned}
$$


and giving profits:

$$
\begin{aligned}
& \pi=\frac{1}{4}(1+\alpha) Q_{H}^{2}-\alpha \gamma Q_{H}+\frac{\alpha^{2} \gamma^{2}}{(1+\alpha)} \text { if } \alpha \gamma>-\frac{1}{2} Q_{H}(1+\alpha), \\
& \pi=-2 \alpha \gamma Q_{H} \text { if } \alpha \gamma<-\frac{1}{2} Q_{H}(1+\alpha) .
\end{aligned}
$$

The profits under the weakest form of truthful peer evaluation $\left(\gamma<\frac{(1+\alpha)|\alpha| Q_{H}}{3+\alpha}\right)$ are described by:

$$
\pi=\frac{1}{4}(1+\alpha) Q_{H}^{2}+\frac{(1-\alpha) \gamma}{2|a|} Q_{H}+\frac{(1-\alpha)^{2} \gamma^{2}}{4(1+\alpha)|a|^{2}}
$$

which are strictly higher than the profits under $m_{i j}=0$ with $\alpha \gamma>-\frac{1}{2} Q_{H}(1+$ $\alpha)$ :

$$
\begin{aligned}
\pi\left(m_{i j}\right. & \left.=s_{i j}\right)-\pi\left(m_{i j}=0\right)=\frac{(1-\alpha) \gamma}{2|a|} Q_{H}+\frac{(1-\alpha)^{2} \gamma^{2}}{4(1+\alpha)|a|^{2}}+\alpha \gamma Q_{H}-\frac{\alpha^{2} \gamma^{2}}{(1+\alpha)}>0 \\
& \Rightarrow\left[\frac{1}{2}(1-\alpha) \frac{\gamma}{|\alpha|}+\alpha \gamma\right] Q_{H}+\frac{1}{(1+\alpha)}\left[\left(\frac{1}{2}(1-\alpha) \frac{\gamma}{|\alpha|}\right)^{2}-(\alpha \gamma)^{2}\right]>0 \\
& \Rightarrow \frac{1}{2}(1-\alpha) \frac{\gamma}{|\alpha|}>-\alpha \gamma \Rightarrow \gamma[1-\alpha+2 \alpha|\alpha|]>0 \Rightarrow 1-\alpha+2 \alpha|\alpha|>0 \\
& \Rightarrow \alpha>-1,
\end{aligned}
$$

which is satisfied by assumption. In case $\alpha \gamma<-\frac{1}{2} Q_{H}(1+\alpha)$, the profits for non-truthful peer evaluation can be higher than those under truthful peer evaluation. The principal does not have to pay any wages in this case. He exploits the bad relationship between the agents by awarding a high peer evaluation bonus, which he never has to pay. Agents exert effort to increase their disliked colleague's lying costs. For extreme lying costs, the principal can obtain high output with certainty for free.

Comparative statics for expected profits and expected utility The expected profits under truthful peer evaluation can be written as:

$$
E(\pi)=2[(1+\alpha) \Delta w+B]\left[Q_{H}-2 \Delta w-B\right] .
$$

By substituting the optimal contract into (B1) gives:

$$
E(\pi)=\frac{1}{2} Q_{H}^{2},
$$


for contract (A), leading to the comparative static effects :

$$
\frac{\partial E(\pi)}{\partial \gamma}=\frac{\partial E(\pi)}{\partial \alpha}=0
$$

Similar, for contract (B) we obtain:

$$
E(\pi)=2 \frac{\gamma}{|\alpha|}\left[Q_{H}-\frac{\gamma}{|\alpha|}\right]
$$

and:

$$
\begin{aligned}
& \frac{\partial E(\pi)}{\partial \gamma}=\frac{2}{|\alpha|}\left[Q_{H}-2 \frac{\gamma}{|\alpha|}\right]>0, \\
& \frac{\partial E(\pi)}{\partial \alpha}=-\frac{2 \alpha \gamma}{|\alpha|^{3}}\left[Q_{H}-2 \frac{\gamma}{|\alpha|}\right],
\end{aligned}
$$

as comparative static results. Expected profits are increasing in $\gamma$ for contract (B), as $\frac{\gamma}{|\alpha|}<\frac{1}{2} Q_{H}$. Likewise, expected profits are increasing in $\alpha$ for $\alpha<0$, and decreasing in $\alpha$ for $\alpha>0$, i.e., expected profits decrease with more pronounced co-worker relation. The expected profits under contract (C) are:

$$
E(\pi)=2\left[\frac{(1+\alpha) Q_{H}}{4}+\frac{(1-\alpha) \gamma}{4|a|}\right]\left[\frac{Q_{H}}{2}+\frac{(1-\alpha) \gamma}{2(1+\alpha)|a|}\right],
$$

which gives comparative static results:

$$
\begin{aligned}
& \frac{\partial E(\pi)}{\partial \gamma}=\frac{(1-\alpha) Q_{H}}{2|a|}+\frac{(1-\alpha)^{2} \gamma}{2(1+\alpha)|a|^{2}}>0, \\
& \frac{\partial E(\pi)}{\partial \alpha}=-\frac{\alpha \gamma}{|\alpha|^{3}}\left[\frac{(1-\alpha) Q_{H}}{2}+\frac{(1-\alpha)^{2} \gamma}{2(1+\alpha)|\alpha|}\right]+2 \Delta w\left[Q_{H}-2 \Delta w-B\right] .
\end{aligned}
$$

Expected profits are increasing in $\gamma$, as lying costs shift compensation towards a higher peer evaluation bonus, which is a more cost-effective manner of stimulating effort. Co-worker relations have two effects on profits: First, as before, co-worker relations affect expected profits through a change in the peer evaluation bonus. Second, better co-worker relations increase the effectiveness of the team bonus. Expected profits are increasing in $\alpha$, except for moderately positive co-worker relations, as the effect through the peer evaluation bonus dominates the favorable effect on the free-rider problem for a small team bonus. 
The comparative static effects with respect to expected utility are best shown when we rewrite (3) slightly. A substitution of $U_{j}$ into $i$ 's utility function, in combination with the homogeneity assumption, gives:

$$
\begin{aligned}
E(U) & =\frac{1}{1-\alpha}[\varphi(\cdot) \Delta w+p(\cdot) B-C(\cdot)] \\
& =\frac{1}{1-\alpha}\left[\frac{1}{2} B^{2}+2 B \Delta w+\frac{1}{2}(3-\alpha)(1+\alpha) \Delta w^{2}\right] .
\end{aligned}
$$

The expected utility under contract (A) is:

$$
E(U)=\frac{1}{1-\alpha} \frac{Q_{H}^{2}}{8},
$$

which yields the following comparative statics:

$$
\begin{aligned}
& \frac{\partial E(U)}{\partial \gamma}=0, \\
& \frac{\partial E(U)}{\partial \alpha}=\frac{1}{(1-\alpha)^{2}} \frac{Q_{H}^{2}}{8}>0 .
\end{aligned}
$$

Contract (B) gives expected utility:

$$
E(U)=\frac{1}{1-\alpha} \frac{1}{2} \frac{\gamma^{2}}{|\alpha|^{2}},
$$

and comparative statics:

$$
\begin{aligned}
& \frac{\partial E(U)}{\partial \gamma}=\frac{\gamma}{(1-\alpha)|\alpha|^{2}}>0 \\
& \frac{\partial E(U)}{\partial \alpha}=\frac{\gamma^{2}}{(1-\alpha)|\alpha|^{2}}\left[\frac{1}{2(1-\alpha)}-\frac{\alpha}{|\alpha|^{2}}\right] .
\end{aligned}
$$

In contract (B), the expected utility is increasing in $\alpha$, except for $0<\alpha<\frac{2}{3}$. Finally, the expected utility in contract $(\mathrm{C})$ equals:

$$
\begin{aligned}
E(U) & =\frac{1}{1-\alpha}\left[\frac{1}{2} B^{2}+2 B \Delta w+\frac{1}{2}(3-\alpha)(1+\alpha) \Delta w^{2}\right] \\
& =\frac{1}{32(1-\alpha)}\left[(3-\alpha) Q_{H}-\frac{(5+\alpha)(1-\alpha) \gamma}{(1+\alpha)|\alpha|}\right]\left[(1+\alpha) Q_{H}+\frac{(1-\alpha) \gamma}{|\alpha|}\right]
\end{aligned}
$$


and comparative static analysis gives:

$$
\begin{aligned}
\frac{\partial E(U)}{\partial \gamma}= & -\frac{1}{1-\alpha} \frac{\partial B}{\partial \gamma}\left[\frac{(1-\alpha) B}{2(1+\alpha)}+\frac{\left(1-\alpha^{2}\right) \Delta w}{4}\right]<0 \\
\frac{\partial E(U)}{\partial \alpha}= & -\frac{1}{1-\alpha} \frac{\partial B}{\partial \alpha}\left[\frac{(1-\alpha) B}{2(1+\alpha)}+\frac{\left(1-\alpha^{2}\right) \Delta w}{4}\right]+\frac{B[(3-\alpha)(1+\alpha) \Delta w+2 B]}{2(1-\alpha)^{2}} \\
& +\frac{1}{(1-\alpha)^{2}}\left[\frac{1}{2} B^{2}+2 B \Delta w+\frac{1}{2}(3-\alpha)(1+\alpha) \Delta w^{2}\right]
\end{aligned}
$$

Expected utility is decreasing in the lying costs. Although higher lying costs allow for a higher peer evaluation bonus, which increases rents as we saw in contract (B), the team bonus decreases simultaneously, which has a stronger effect on worker's rents. There is a similar effect on utility for co-worker relations. As these become more pronounced, compensation shifts towards a higher team bonus, denoted by the first term. This term is negative for $\alpha<0$ and positive for $\alpha>0$. Further, utility increases in the co-worker relations through a softened free-rider problem and the direct effect on utility, captured by the second and third term respectively. In sum, utility is increasing in $\alpha$, excepts for some moderately negative co-worker relations, where the first term dominates. 


\section{References}

[1] Antonioni, David (1996), Designing an Effective 360-Degree Appraisal Feedback Process, Organizational Dynamics, Vol. 25(2): $24-38$.

[2] Barron, John M. and Manish M. Gupte (2009), Limits to Peer Appraisals: Peer Pressure and Team Cohesion, mimeo, Purdue University.

[3] Bohl, Don L. (1996), Minisurvey: 360-degree appraisals yield superior results, survey shows, Compensation and Benefits Review, Vol. 28(5): $16-19$.

[4] Carmichael, Lorne H. (1988), Incentives in Academics: Why is There Tenure?, Journal of Political Economy, Vol. 96(3): 453 - 472.

[5] Carpenter, Jeffrey, Peter H. Matthews, and John Schirm (2010), Tournaments and Office Politics: Evidence from a Real Effort Experiment, American Economics Review Vol 100(1): 504 - 517.

[6] Coates, Dennis E. (1998), Don’t Tie 360 Feedback to Pay, Training, Vol $35(9): 68-78$.

[7] Dur, Robert and Joeri Sol (2010), Social Interaction, Co-Worker Altruism, and Incentives, forthcoming in Games and Economic Behavior.

[8] Edwards, Mark R. and Ann J. Ewen (1996), How to Manage Performance and Pay With 360-Degree Feedback, Compensation and Benefits Review, Vol 28(3): $41-46$.

[9] Fischbacher, Urs and Franziska Heusi (2008), Lies in Disguise. An experimental study on cheating, TWI Working Paper 40.

[10] Fischer, Paul and John S. Hughes (1997), Mutual Monitoring and Best Agency Contracts, Journal of Institutional and Theoretical Economics, Vol 153(2): $334-355$.

[11] Fleenor, John W. and Stéphane Brutus (2001), Multisource Feedback for Personnel Decisions, in: David Bracken, Carol W. Timmreck, Allan H. Church (Eds.), The Handbook of Multisource Feedback, San Francisco, CA: Jossey-Bass. 
[12] Freeman, Richard B., Douglas Kruse, and Joseph Blasi (2008), Workers' Response to Shirking Under Shared Capitalism, NBER Working Paper Number 14227.

[13] Gneezy, Uri (2005), Deception: The Role of Consequences, American Economic Review, Vol. 95(1): 384 - 394.

[14] Gupte, Manish M. (2009), Developmental Peer Appraisals, mimeo, Purdue University.

[15] Holmstrom, Bengt (1982), Moral Hazard in Teams, Bell Journal of Economics, Vol 13(2): $324-340$.

[16] Hurkens, Sjaak and Navin Kartik (2009), Would I Lie to You? On Social Preferences and Lying Aversion, Experimental Economics, Vol. 12(2): $180-192$.

[17] Kartik, Navin (2009), Strategic communication with lying costs, Review of Economic Studies, Vol. 76(4): 1359 - 1395

[18] Kozlowski, Steve W. J., Georgia T. Chao, and Robert F. Morrison (1998), Games raters play: Politics, strategies, and impression management in performance appraisal, in: James W. Smither (Ed.), Performance appraisal: State of the art in practice, San Francisco: JosseyBass.

[19] Ma, Ching-To (1988), Unique Implementation of Incentive Contracts with Many Agents, Review of Economic Studies, Vol 55(4): 555 - 571.

[20] Marx, Leslie M. and Francesco Squintani (2009), Individual Accountability in Teams with Adverse Selection, Journal of Economic Behavior E3 Organization, Vol. 72(1): 260-273.

[21] Peiperl, Maury A. (1999), Conditions for the success of peer evaluations, International Journal of Human Resource Management, Vol 10(3): 429 $-458$.

[22] Towry, Kristy L. (2003), Control in a Teamwork Environment: The Impact of Social Ties on the Effectiveness of Mutual Monitoring Contracts, Accounting Review, Vol. 78(4): 1069 - 1095. 